\title{
Article
}

\section{On (2-d)-Kernels in Two Generalizations of the Petersen Graph}

\author{
Paweł Bednarz ${ }^{*}+\mathbb{D}$ and Natalia Paja ${ }^{+} \mathbb{D}$ \\ The Faculty of Mathematics and Applied Physics, Rzeszów University of Technology, \\ al. Powstańców Warszawy 12, 35-959 Rzeszów, Poland; nbednarz@prz.edu.pl \\ * Correspondence: pbednarz@prz.edu.pl \\ + These authors contributed equally to this work.
}

check for updates

Citation: Bednarz, P.; Paja, N. On (2-d)-Kernels in Two Generalizations of the Petersen Graph. Symmetry 2021, 13, 1948. https://doi.org/10.3390/ sym13101948

Academic Editors: Markus Meringer and Juan Alberto Rodríguez Velázquez

Received: 5 August 2021

Accepted: 7 October 2021

Published: 16 October 2021

Publisher's Note: MDPI stays neutral with regard to jurisdictional claims in published maps and institutional affiliations.
Abstract: A subset $J$ is a (2- $d)$-kernel of a graph if $J$ is independent and 2-dominating simultaneously. In this paper, we consider two different generalizations of the Petersen graph and we give complete characterizations of these graphs which have $(2-d)$-kernel. Moreover, we determine the number of $(2-d)$-kernels of these graphs as well as their lower and upper kernel number. The property that each of the considered generalizations of the Petersen graph has a symmetric structure is useful in finding (2-d)-kernels in these graphs.

Keywords: domination; independence; (2- $d)$-kernel; generalized Petersen graphs

\section{Introduction}

In general, we use the standard terminology and notation of graph theory (see [1]). Let $G$ be an undirected, connected, and simple graph with the vertex set $V(G)$ and the edge set $E(G)$. The order of the graph $G$ is the number of vertices in $G$. The size of the graph $G$ is its number of edges. By $P_{n}, n \geq 1$ and $C_{n}, n \geq 3$, we mean a path and a cycle of order $n$, respectively.

Let $G=(V, E)$ and $G^{\prime}=\left(V^{\prime}, E^{\prime}\right)$ be two graphs. If $V^{\prime} \subseteq V$ and $E^{\prime} \subseteq E$, then $G^{\prime}$ is a subgraph of $G$, written as $G^{\prime} \subseteq G$. If $G^{\prime} \subseteq G$ and $G^{\prime}$ contain all the edges $x y \in E$ with $x, y \in V^{\prime}$, then $G^{\prime}$ is an induced subgraph of $G$ and we write $G^{\prime}:=\left\langle V^{\prime}\right\rangle_{G}$. Graphs $G$ and $G^{\prime}$ are called isomorphic, and denoted by $G \cong G^{\prime}$, if there exists a bijection $\phi: V \rightarrow V^{\prime}$ with $x y \in E \Leftrightarrow \phi(x) \phi(y) \in E^{\prime}$ for all $x, y \in V$. The complement of the graph $G$ is a graph $\bar{G}$ such that $V(G)=V(\bar{G})$ and two distinct vertices of $\bar{G}$ are adjacent if and only if they are not adjacent in $G$. A graph $G$ is called bipartite if $V(G)$ admits a partition into two classes such that every edge has its ends in different classes.

A subset $D \subseteq V(G)$ is a dominating set of $G$ if each vertex of $G$ not belonging to $D$ is adjacent to at least one vertex of $D$. A subset $S \subseteq V(G)$ is called an independent set of $G$ if no two vertices of $S$ are adjacent in $G$. A subset $J$ being independent and dominating is a kernel of $G$.

The concept of kernels was initiated in 1953 by von Neumann and Morgenstern in digraphs with regard to game theory (see [2]). One of the pioneers studying the kernels in digraphs was C. Berge (see [3-5]). In literature, we can find many types and generalizations of kernels in digraphs (for results and applications, see, for example, [6-11]). The problem of the existence of kernels in undirected graphs is trivial because every maximal independent set is a kernel. Currently, distinct kind of kernels in undirected graphs are being studied quite intensively and many papers are available. For results and application, see, for example, [12-18]. Among many types of kernels in undirected graphs, there are kernels related to multiple domination, introduced by Fink and Jacobson in [19]. Let $p \geq 1$ be an integer. A subset $S$ is said to be $p$-dominating if every vertex outside $S$ has at least $p$ neighbors in $S$. If $p=1$, then we obtain a dominating set in the classical sense. If $p=2$, we get a 2-dominating set. A set which is 2-dominating and independent is named a 2dominating kernel ((2- $d)$-kernel in short). The concept of $(2-d)$-kernels was introduced by 
A. Włoch in [20]. Some properties of (2-d)-kernels were studied in [21-24]. In particular, in [23], it was proved that the problem of the existence of $(2-d)$-kernels is $\mathcal{N P}$-complete for general graphs. In [25], Nagy extended the concept of $(2-d)$-kernels to $k$-dominating kernels. He considered a $k$-dominating set instead of the 2-dominating set, which he called $k$-dominating independent sets. Some properties of these sets were studied in $[26,27]$.

The number of $(2-d)$-kernels in the graph $G$ is denoted by $\sigma(G)$. Let $G$ be a graph with the $(2-d)$-kernel. The minimum cardinality of the $(2-d)$-kernel of $G$ is called a lower $(2-d)$ kernel number and denoted by $\gamma_{(2-d)}(G)$. The maximum cardinality of the $(2-d)$-kernel of $G$ is called an upper $(2-d)$-kernel number and is denoted by $\Gamma_{(2-d)}(G)$.

In this paper, we consider two different generalizations of the Petersen graph. Various types of domination in the class of generalized Petersen graphs have been extensively studied in the literature (see [28-32]). Referring to this research, we will consider (2- $d$ )kernels for two different generalizations of the Petersen graph. We solve the problem of the existence of $(2-d)$-kernels, their number, and their cardinality in these graphs. Moreover, we determine a lower and an upper kernel number in these graphs. It is worth noting that each of presented generalizations of the Petersen graph has a symmetric structure. This property is useful in finding $(2-d)$-kernels in these graphs.

\section{Main Results}

In this section, we consider the problem of the existence of $(2-d)$-kernels in two different generalizations of the Petersen graph. In particular, we give complete characterizations of these generalizations, which have the $(2-d)$-kernel. We determine the number of $(2-d)$-kernels in these graphs as well as the lower and the upper $(2-d)$-kernel number.

In the further part of the paper, we will use green color to mark vertices belonging to the (2- $d)$-kernel, and red color to indicate vertices that cannot belong to it.

\subsection{Generalized Petersen Graph}

Let $n \geq 3, k<\frac{n}{2}$ be integers. The graph $P(n, k)$ is called the generalized Petersen graph, if $V(P(n, k))=\bigcup_{i=0}^{n-1}\left\{u_{i}, v_{i}\right\}$ and $E(P(n, k))=\bigcup_{i=0}^{n-1}\left\{u_{i} u_{i+1}, u_{i} v_{i}, v_{i} v_{i+k}\right\}$, where subscripts are reduced modulo $n$. These graphs were first defined by Watkins in [33]. Figure 1 shows generalized Petersen graphs $P(10,3), P(5,2)$ and examples of $(2-d)$-kernels in these graphs.
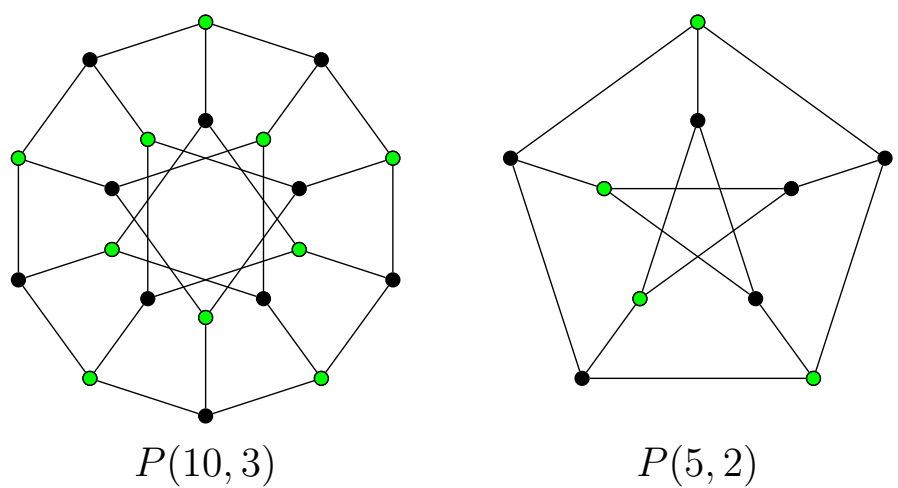

Figure 1. Examples of $(2-d)$-kernels in $P(10,3)$ and $P(5,2)$.

We start with the problem of existence of $(2-d)$-kernels. At the beginning, we give a sufficient condition, emerging from the property of bipartite graphs. We have the following complete characterization of bipartite generalized Petersen graphs.

Proposition 1 ([34]). Let $n \geq 3, k<\frac{n}{2}$ be integers. The graph $P(n, k)$ is bipartite if and only if $n$ is even and $k$ is odd. 
From this characterization we directly obtain the sufficient condition for the existence of (2-d)-kernels.

Proposition 2. Let $n \geq 3, k<\frac{n}{2}$ be integers. If $n$ is even and $k$ is odd, then the graph $P(n, k)$ has at least two (2-d)-kernels which are a partition of the vertex set.

Proof. Let $n, k$ be as in the statement of the proposition. From Proposition 1 , it follows that the graph $P(n, k)$ is a bipartite graph. Thus, there exist two independent sets of vertices $V_{1}, V_{2}$ that are a partition of the set $V(P(n, k))$. Moreover, the graph $P(n, k)$ is a 3-regular graph. Therefore, sets $V_{1}, V_{2}$ are $(2-d)$-kernels of the graph $P(n, k)$.

Now, we improve the above proposition to obtain the complete characterization of the generalized Petersen graph having $(2-d)$-kernel.

Theorem 1. Let $n \geq 3, k<\frac{n}{2}$ be integers. The graph $P(n, k)$ has a (2-d)-kernel if and only if

$$
n \text { is even and } k \text { is odd or }
$$

(ii) $\quad n \equiv 0(\bmod 5)$ and $k \equiv 2(\bmod 5)$ or

(iii) $n \equiv 0(\bmod 5)$ and $k \equiv 3(\bmod 5)$.

Proof. If $n=3,4$, then the result is obvious. Let $n \geq 5, k<\frac{n}{2}$ be integers. If $n$ is even and $k$ is odd, then by Proposition 2, (i) follows. Let $n \equiv 0(\bmod 5), k \equiv j(\bmod 5), j=2,3$. We will show that the set $J=\left\{u_{i} ; i \in\{0,5, \ldots, n\}\right\} \cup\left\{u_{i+2} ; i \in\{0,5, \ldots, n\}\right\} \cup\left\{v_{i+3} ; i \in\right.$ $\{0,5, \ldots, n\}\} \cup\left\{v_{i+4} ; i \in\{0,5, \ldots, n\}\right\}$ is a $(2-d)$-kernel of $P(n, k)$. The independence of $J$ follows from the definition of $P(n, k)$. Let us assume that $x \in V(P(n, k)) \backslash J$. Then, either $x=u_{s}, s \in\{0,1, \ldots, n-1\}, s \equiv a(\bmod 5), a=1,3,4$ or $x=v_{t}, t \in\{0,1, \ldots, n-1\}$, $t \equiv b(\bmod 5), b=0,1,2$. We consider two cases.

1. $x=u_{s}$.

If $s \equiv 1(\bmod 5)$, then $\left\{u_{s-1}, u_{s+1}\right\} \subseteq N\left(u_{s}\right)$ and $u_{s-1}, u_{s+1} \in J$. If $s \equiv 3(\bmod 5)$, then $\left\{u_{s-1}, v_{s}\right\} \subseteq N\left(u_{s}\right)$ and $u_{s-1}, v_{s} \in J$. If $s \equiv 4(\bmod 5)$, then $\left\{u_{s+1}, v_{s}\right\} \subseteq N\left(u_{s}\right)$ and $u_{s+1}, v_{s} \in J$.

2. $x=v_{t}$.

Let $t \equiv 0(\bmod 5)$. If $k \equiv 2(\bmod 5)$, then $\left\{u_{t}, v_{t-2}\right\} \subseteq N\left(v_{t}\right)$ and $u_{t}, v_{t-2} \in J$. If $k \equiv$ $3(\bmod 5)$, then $\left\{u_{t}, v_{t+3}\right\} \subseteq N\left(v_{t}\right)$ and $u_{t}, v_{t+3} \in J$. If $t \equiv 1(\bmod 5)$, then $\left\{v_{t-k}, v_{t+k}\right\} \subseteq$ $N\left(v_{t}\right)$ and $v_{t-k}, v_{t+k} \in J, k \equiv j(\bmod 5), j=2,3$. Let $t \equiv 2(\bmod 5)$. If $k \equiv 2(\bmod 5)$, then $\left\{u_{t}, v_{t+2}\right\} \subseteq N\left(v_{t}\right)$ and $u_{t}, v_{t+2} \in J$. If $k \equiv 3(\bmod 5)$, then $\left\{u_{t}, v_{t-3}\right\} \subseteq N\left(v_{t}\right)$ and $u_{t}, v_{t-3} \in J$.

Summing up all the above cases we obtain that every vertex $x \in V(P(n, k)) \backslash J$ is 2-dominated by $J$. Hence, $J$ is a $(2-d)$-kernel of $P(n, k)$.

Conversely, let $n \geq 5, k<\frac{n}{2}, i \in\{0,1, \ldots, n-1\}$ be integers and let $J$ be a $(2-d)$-kernel of $P(n, k)$. If $u_{i}, u_{i+1}, u_{i+2} \notin J$, then the vertex $u_{i+1}$ is not 2 -dominated by $J$. Thus, each connected component of the graph $\left\langle\bigcup_{i=0}^{n-1} u_{i}\right\rangle_{P(n, k)} \backslash J$ is isomorphic to either $P_{1}$ or $P_{2}$. We will show that in the graph $P(n, k)$ having a $(2-d)$-kernel, the configurations of these paths $P_{1}, P_{2}$ on the outer cycle, which are shown in the Figure 2 are forbidden.
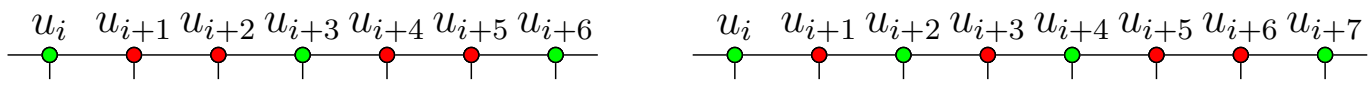

Figure 2. Forbidden configurations of the paths $P_{1}, P_{2}$ for the graph $P(n, k)$ with the $(2-d)$-kernel.

Let us consider the following cases.

1. First, we will prove that the configuration of the paths $P_{1}, P_{2}$ shown on the left side of the Figure 2 is forbidden. Suppose that $u_{i}, u_{i+3}, u_{i+6} \in J$ for some $i$, as in Figure 3 . Then, 
$v_{i+1}, v_{i+2}, v_{i+4}, v_{i+5} \in J$; otherwise, vertices $u_{i+1}, u_{i+2}, u_{i+4}, u_{i+5}$ are not 2-dominated by $J$. Therefore, for every $k$ vertices $v_{i+1+k}, v_{i+2+k}, v_{i+4+k}, v_{i+5+k} \notin J$.
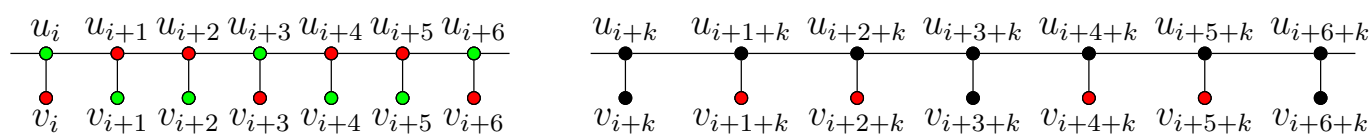

Figure 3. The case when $u_{i}, u_{i+3}, u_{i+6} \in J$.

We have the next two possibilities.

1.1. $v_{i+3+k} \notin J$ for some $i$ (see Figure 4).

Since $v_{i+3+k} \notin J$, the vertex $u_{i+3+k} \in J$ and $u_{i+2+k}, u_{i+4+k} \notin J$. Then $v_{i+2+2 k}, v_{i+3+2 k}$, $v_{i+4+2 k} \in J$. This means that $u_{i+2+2 k}, u_{i+3+2 k}, u_{i+4+2 k} \notin J$. Hence, the vertex $u_{i+3+2 k}$ is not 2-dominated by $J$, a contradiction.

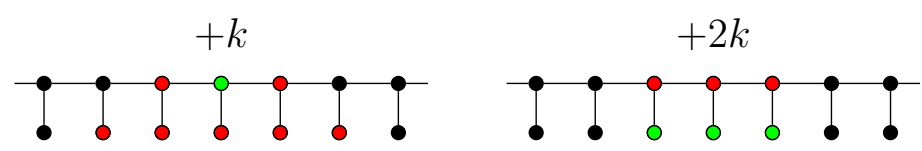

Figure 4. The case when $u_{i}, u_{i+3}, u_{i+6} \in J$ (the first subcase).

1.2. $v_{i+3+k} \in J$ for some $i$ (see Figure 5).

Then, $u_{i+3+k} \notin J$ and $u_{i+2+k}, u_{i+4+k} \in J$; otherwise, they are not 2-dominated by $J$. Because $J$ is an independent set, $u_{i+1+k}, u_{i+5+k} \notin J$. Moreover, $u_{i+k}, u_{i+6+k} \in J$ to 2dominate $u_{i+1+k}, u_{i+5+k}$. Hence, $v_{i+k}, v_{i+6+k} \notin J$. To 2-dominate $v_{i+1+k}, v_{i+5+k}$, we must have $v_{i+1+2 k}, v_{i+5+2 k} \in J$. Moreover, $u_{i+1+2 k}, u_{i+5+2 k}, v_{i+3+2 k} \notin J$. Since $v_{i+k}, v_{i+6+k}$ have exactly one neighbour in $J$, vertices $v_{i+2 k}, v_{i+6+2 k} \in J$ and $u_{i+2 k}, u_{i+6+2 k} \notin J$. Next, $u_{i+2+2 k}, u_{i+4+2 k} \in J$ to 2 -dominate $u_{i+1+2 k}, u_{i+5+2 k}$ and $v_{i+2+2 k}, v_{i+4+2 k}, u_{i+3+2 k} \notin J$. Thus, $v_{i+2+3 k}, v_{i+3+3 k}, v_{i+4+3 k} \in J$ to 2 -dominate $v_{i+2+2 k}, v_{i+3+2 k}, v_{i+4+2 k}$. Therefore, $u_{i+2+3 k}$, $u_{i+3+3 k}, u_{i+4+3 k} \notin J$. This means that $u_{i+3+3 k}$ is not 2 -dominated, a contradiction.
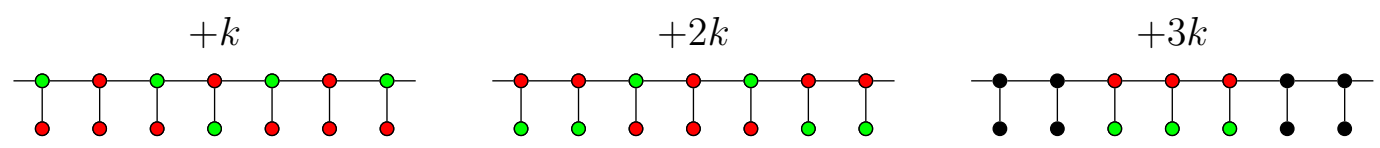

Figure 5. The case when $u_{i}, u_{i+3}, u_{i+6} \in J$ (the second subcase).

Hence, for each $n$ and $k$, it is not possible that the vertices $u_{i}, u_{i+3}, u_{i+6}$ belong to a (2-d)-kernel of $P(n, k)$.

2. Now, we will prove that the configuration of the paths $P_{1}, P_{2}$ shown on the right side of the Figure 2 is forbidden. Suppose that $u_{i}, u_{i+2}, u_{i+4}, u_{i+7} \in J$ for some $i$, as in Figure 6 . Then, $v_{i+5}, v_{i+6}$, which causes $v_{i}, v_{i+1}, v_{i+2}, v_{i+3}, v_{i+4}, v_{i+5}, v_{i+6}, v_{i+7} \notin J$.

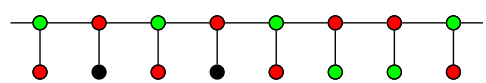

Figure 6. The case when $u_{i}, u_{i+2}, u_{i+4}, u_{i+7} \in J$.

We consider four subcases.

2.1. $v_{i+1}, v_{i+3} \notin J$ for some $i$ (see Figure 7).

Then, $v_{i+1-k}, v_{i+3-k}, v_{i+1+k}, v_{i+3+k} \in J$. Since $v_{i+2}$ must be 2-dominated, so $v_{i+2-k} \in J$ or $v_{i+2+k} \in J$. Without loss of generality, assume that $v_{i+2+k} \in J$. Thus, $u_{i+1+k}, u_{i+2+k}$, $u_{i+3+k} \notin J$. Hence, the vertex $u_{i+2+k}$ is not 2 -dominated, a contradiction.

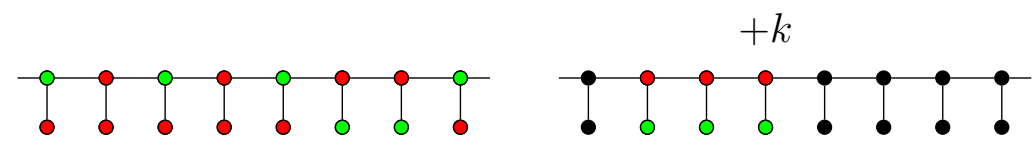

Figure 7. The case when $u_{i}, u_{i+2}, u_{i+4}, u_{i+7} \in J$ (the first subcase). 
2.2. $v_{i+1} \notin J$ and $v_{i+3} \in J$ for some $i$ (see Figure 8).

Then, $v_{i+3+k}, v_{i+5+k}, v_{i+6+k} \notin J$. Since $v_{i+7}$ must be 2 -dominated, we obtain that $v_{i+7-k} \in J$ or $v_{i+7+k} \in J$. Without loss of generality, assume that $v_{i+7+k} \in J$. Thus, $u_{i+7+k} \notin J$ and $u_{i+6+k} \in J$. Because $J$ is an independent set and $u_{i+6+k} \in J, u_{i+5+k} \notin J$. Therefore, $u_{i+4+k} \in J$, which causes $u_{i+3+k}, v_{i+4+k} \notin J$. Moreover, $v_{i+3+2 k}, v_{i+4+2 k}, v_{i+5+2 k} \in J$, and finally $u_{i+3+2 k}, u_{i+4+2 k}, u_{i+5+2 k} \notin J$. Hence, the vertex $u_{i+4+2 k}$ is not 2 -dominated, a contradiction.

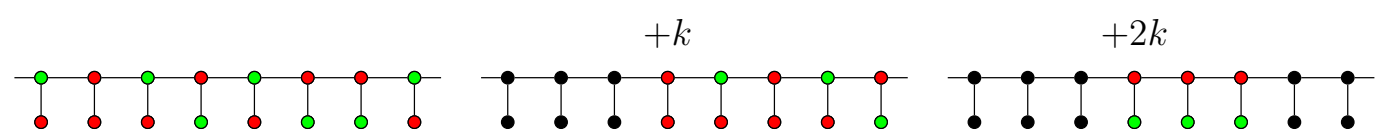

Figure 8. The case when $u_{i}, u_{i+2}, u_{i+4}, u_{i+7} \in J$ (the second subcase).

2.3. $v_{i+1} \in J$ and $v_{i+3} \notin J$ for some $i$ (see Figure 9).

Then, $v_{i+3+k} \in J$ and $v_{i+1+k}, v_{i+5+k}, v_{i+6+k}, u_{i+3+k} \notin J$. Since $v_{i+4}$ must be 2-dominated, $v_{i+4-k} \in J$ or $v_{i+4+k} \in J$. Without loss of generality, assume that $v_{i+4+k} \in J$. Thus, $u_{i+4+k} \notin J$. Moreover, $u_{i+2+k}, u_{i+5+k} \in J$, which causes $u_{i+1+k}, u_{i+6+k}, v_{i+2+k} \notin J$. To 2-dominate $u_{i+1+k}$, we must have $u_{i+k} \in J$. Then, $v_{i+k} \notin J$ and $v_{i+2 k}, v_{i+1+2 k}, v_{i+2+2 k} \in J$. From the independence of the set $J$, we get that $u_{i+2 k}, u_{i+1+2 k}, u_{i+2+2 k} \notin J$. Hence, the vertex $u_{i+1+2 k}$ is not 2 -dominated, a contradiction.

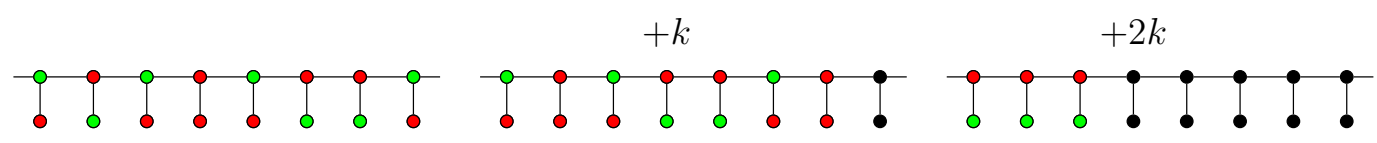

Figure 9. The case when $u_{i}, u_{i+2}, u_{i+4}, u_{i+7} \in J$ (the third subcase).

2.4. $v_{i+1}, v_{i+3} \in J$ for some $i$.

Proving analogously as in subcase 2.3., we obtain a contradiction with the assumption that $J$ is a $(2-d)$-kernel.

Therefore, for each $n$ and $k$, it is not possible that the vertices $u_{i}, u_{i+2}, u_{i+4}, u_{i+7}$ belong to a (2-d)-kernel of $P(n, k)$.

Hence, for the graph with the (2- $d)$-kernel, the configurations of $P_{1}, P_{2}$ shown in the Figure 10 are the only ones that may be possible. Now, we will show that they are indeed possible.

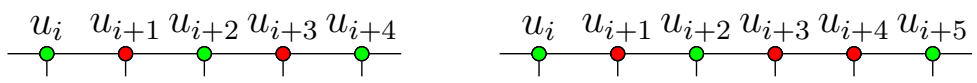

Figure 10. Possible configurations of the paths $P_{1}, P_{2}$ for the graph $P(n, k)$ with the $(2-d)$-kernel.

3. Suppose that $u_{i}, u_{i+2}, u_{i+4} \in J$ for some $i$, as in Figure 11. Then, $u_{i+1}, u_{i+3}, v_{i}, v_{i+2}$, $v_{i+4} \notin J$.

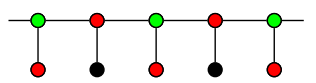

Figure 11. The case when $u_{i}, u_{i+2}, u_{i+4} \in J$.

We consider four subcases.

3.1. $v_{i+1}, v_{i+3} \notin J$ for some $i$ (see Figure 12).

Since $v_{i+2}$ must be 2-dominated, we obtain that $v_{i+2+k} \in J$ or $v_{i+2-k} \in J$. Without loss of generality, assume that $v_{i+2+k} \in J$. Moreover, $v_{i+1+k}, v_{i+3+k} \in J$ and $u_{i+1+k}, u_{i+2+k}, u_{i+3+k} \notin$ $J$. Hence, the vertex $u_{i+2+k}$ is not 2 -dominated, a contradiction. 


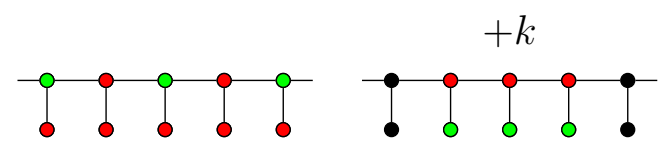

Figure 12. The case when $u_{i}, u_{i+2}, u_{i+4} \in J$ (the first subcase).

3.2. $v_{i+1} \notin J$ and $v_{i+3} \in J$ for some $i$ (see Figure 13).

Then, $v_{i+1+k} \in J$ and $v_{i+3+k} \notin J$. Since $v_{i+2}$ must be 2-dominated, $v_{i+2+k} \in J$ or $v_{i+2-k} \in J$. Without loss of generality, assume that $v_{i+2+k} \in J$. Thus, $u_{i+1+k}, u_{i+2+k} \notin J$ and $u_{i+k}, u_{i+3+k} \in J$, which causes $v_{i+k}, u_{i+4+k} \notin J$ and $v_{i+4+k} \in J$. Moreover, $v_{i+1+2 k}$, $v_{i+2+2 k}, v_{i+4+2 k} \notin J, v_{i+2 k} \in J, u_{i+2 k} \notin J, u_{i+1+2 k} \in J, u_{i+2+2 k} \notin J, u_{i+3+2 k} \in J$ and $u_{i+4+2 k}, v_{i+3+2 k} \notin J$. Finally, $v_{i+2+3 k}, v_{i+3+3 k}, v_{i+4+3 k} \in J$ and $u_{i+2+3 k}, u_{i+3+3 k}, u_{i+4+3 k} \notin J$. Hence, the vertex $u_{i+3+3 k}$ is not 2 -dominated, a contradiction.

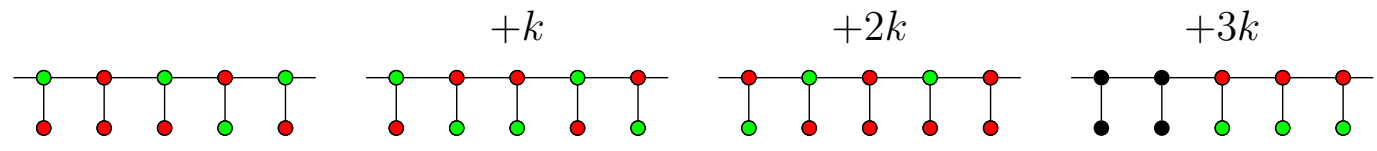

Figure 13. The case when $u_{i}, u_{i+2}, u_{i+4} \in J$ (the second subcase).

3.3. $v_{i+1} \in J$ and $v_{i+3} \notin J$ for some $i$.

Proving analogously as in subcase 3.2., we obtain a contradiction with the assumption that $J$ is a $(2-d)$-kernel.

3.4. $v_{i+1}, v_{i+3} \in J$ for some $i$ (see Figure 14).

Then, $v_{i+1+k}, v_{i+3+k} \notin J$. First, we will show that $v_{i+k}$ and $v_{i-k}$ must belong to a (2-d)kernel $J$. Suppose on contrary that $v_{i+k} \notin J$. Since $v_{i+k}$ must be 2 -dominated, $u_{i+k} \in J$. Thus, $u_{i+1+k} \notin J$ and $u_{i+2+k} \in J$. Moreover, $v_{i+2 k}, v_{i+1+2 k}, v_{i+2+2 k} \in J$ and $u_{i+2 k}, u_{i+1+2 k}$, $u_{i+2+2 k} \in J$. Hence, the vertex $u_{i+1+2 k}$ is not 2 -dominated, a contradiction.

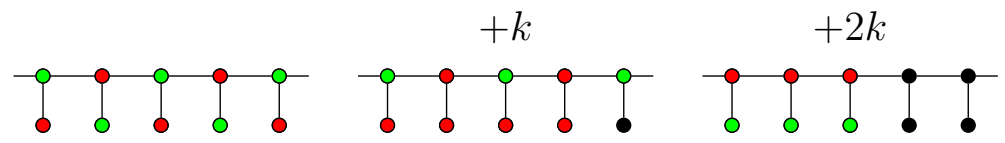

Figure 14. The case when $u_{i}, u_{i+2}, u_{i+4} \in J$ (the fourth subcase).

This means that $v_{i+k}, v_{i-k} \in J$ and also $v_{i+2+k}, v_{i+4+k}, u_{i+1+k}, u_{i+3+k}$ belong to a (2-d)kernel (see Figure 15).

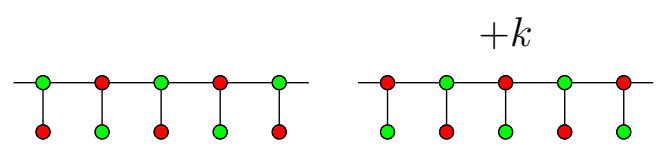

Figure 15. The case when $u_{i}, u_{i+2}, u_{i+4} \in J$ implies that $v_{i+k}, v_{i+2+k}, v_{i+4+k}, u_{i+1+k}, u_{i+3+k} \in J$.

Hence, $n$ must be even, and from the definition of $P(n, k)$, we conclude that $k$ must be odd, which proves (i).

4. Suppose that $u_{i}, u_{i+2}, u_{i+5} \in J$ for some $i$. Then, $u_{i+1}, u_{i+3}, u_{i+4}, v_{i}, v_{i+2}, v_{i+5} \notin J$. Since $u_{i+3}, u_{i+4}$ must be 2-dominated, $v_{i+3}, v_{i+4} \in J$. First, we prove that $v_{i+1} \notin J$. Suppose on contrary that $v_{i+1} \in J$, as in Figure 16. Then, $v_{i+1+k}, v_{i+3+k}, v_{i+4+k} \notin J$. Since $v_{i}$ must be 2-dominated, $v_{i-k} \in J$ or $v_{i+k} \in J$. Without loss of generality, assume that $v_{i+k} \in J$. Thus, $u_{i+k} \notin J, u_{i+1+k} \in J$ and $u_{i+2+k} \notin J$. Moreover, $u_{i+3+k} \in J, u_{i+4+k} \notin J$, $u_{i+5+k} \in J, v_{i+5+k} \notin J$, and $v_{i+2+k} \in J$. Proving analogously as in subcase 3.3., we obtain a contradiction with the assumption that $J$ is a (2-d)-kernel.

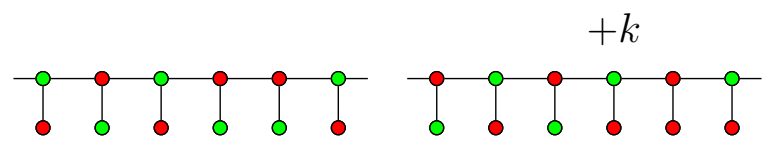

Figure 16. The case when $u_{i}, u_{i+2}, u_{i+5}, v_{i+1} \in J$. 
Hence, $v_{i+1} \notin J$ (see Figure 17). Moreover, $v_{i+1+k} \in J$ and $u_{i+1+k}, v_{i+3+k}, v_{i+4+k} \notin J$. We consider two subcases.

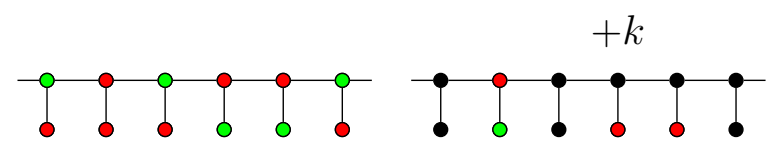

Figure 17. The case when $u_{i}, u_{i+2}, u_{i+5} \in J$, and $v_{i+1} \notin J$.

4.1. $v_{i+2+k} \notin J$ for some $i$ (see Figure 18).

Then, $u_{i+2+k} \in J, u_{i+3+k} \notin J, u_{i+4+k} \in J, u_{i+5+k} \notin J$, and $v_{5+i+k} \in J$. Moreover, $v_{i+k} \in J$ and $u_{i+k} \notin \mathrm{J}$; otherwise, we obtain the same configuration as in subcase 3.3.

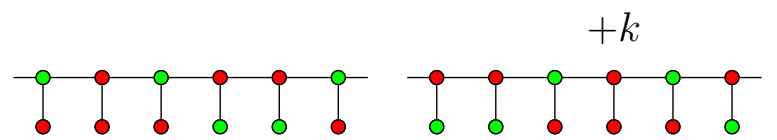

Figure 18. The case when $u_{i}, u_{i+2}, u_{i+5} \in J$ (the first subcase).

Hence, $n$ must be divisible by 5 , and from the definition of $P(n, k)$, we conclude that $k \equiv 2(\bmod 5)$, which proves (ii).

4.2. $\quad v_{i+2+k} \in J$ for some $i$ (see Figure 19).

Then, $u_{i+2+k} \notin J, u_{i+k}, u_{i+3+k} \in J$ and $v_{i+k}, u_{i+4+k} \notin J$. Moreover, $u_{i+5+k} \in J$ and $v_{i+5+k} \notin J$

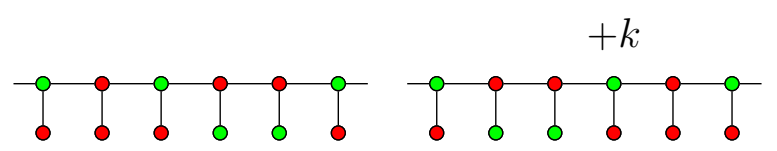

Figure 19. The case when $u_{i}, u_{i+2}, u_{i+5} \in J$ (the second subcase).

Hence, $n$ must be divisible by 5 , and from the definition of $P(n, k)$, we conclude that $k \equiv 3(\bmod 5)$, which proves (iii), which ends the proof.

Basing on the proof of Theorem 1, the following corollaries are obtained. They concern the number of $(2-d)$-kernels in the generalized Petersen graph as well as the lower and upper $(2-d)$-kernel numbers. By a rotation of configurations shown on Figure 10, condition (i) of Theorem 1 gives two (2- $d$ )-kernels in generalized Petersen graph and conditions (ii) and (iii) give five (2- $d$ )-kernels. Therefore, if $n$ and $k$ satisfy more than one of these conditions, we obtain more (2- $d)$-kernels. Moreover, the proof of the Theorem 1 presents the constructions of the (2- $d$ )-kernels in the generalized Petersen graph $P(n, k)$. Figure 20 shows the smallest and the largest $(2-d)$-kernel in the graph $P(20,7)$.

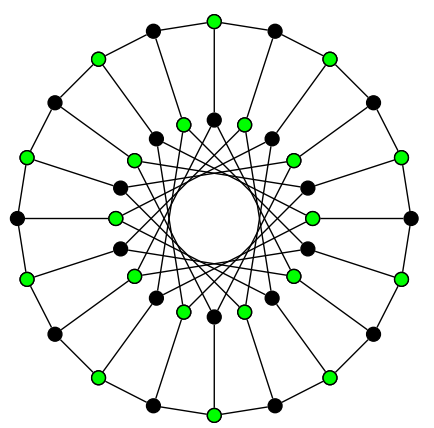

$P(20,7)$

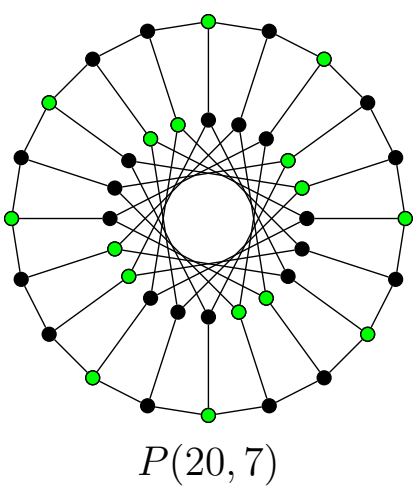

Figure 20. The largest (left side) and the smallest (right side) (2- $d$ )-kernel in the graph $P(20,7)$. 
Corollary 1. Let $n \geq 3, k<\frac{n}{2}$ be integers. Then,

$$
\sigma(P(n, k))=\left\{\begin{array}{cl}
7 & \text { for } n \equiv 0(\bmod 10) \text { and } k \equiv a(\bmod 10), a=3,7 \\
5 & \text { for } n \equiv 5(\bmod 10) \text { and } k \equiv a(\bmod 5), a=2,3 \text { or } \\
& \text { for } n \equiv 0(\bmod 10) \text { and } k \equiv a(\bmod 10), a=2,8 \\
2 & \text { for } n \equiv 0(\bmod 10) \text { and } k \equiv a(\bmod 10), a=1,5,9 \text { or } \\
& \text { for even } n, n \neq 0(\bmod 10) \text { and odd } k .
\end{array}\right.
$$

Corollary 2. Let $n \geq 3, k<\frac{n}{2}$ be integers. If $n \equiv 0(\bmod 10)$ and $k \equiv a(\bmod 10), a=3,7$, then

$$
\gamma_{(2-d)}(P(n, k))=\frac{4}{5} n \quad \text { and } \quad \Gamma_{(2-d)}(P(n, k))=n
$$

Corollary 3. Let $n \geq 3, k<\frac{n}{2}$ be integers. If $n \equiv 5(\bmod 10)$ and $k \equiv a(\bmod 5), a=2,3$ or $n \equiv 0(\bmod 10)$ and $k \equiv a(\bmod 10), a=2,8$, then

$$
\gamma_{(2-d)}(P(n, k))=\Gamma_{(2-d)}(P(n, k))=\frac{4}{5} n
$$

Corollary 4. Let $n \geq 3, k<\frac{n}{2}$ be integers. If $n \equiv 0(\bmod 10)$ and $k \equiv a(\bmod 10), a=1,5,9$ or $n$ is even, $n \not \equiv 0(\bmod 10)$ and $k$ is odd, then

$$
\gamma_{(2-d)}(P(n, k))=\Gamma_{(2-d)}(P(n, k))=n
$$
kernel.

The above corollaries characterize all possible graphs $P(n, k)$, which have the (2- $d)$ -

\subsection{The Second Generalization of the Petersen Graph}

Now, we consider another generalization of the Petersen graph. Let $n \geq 5$ be an integer. Let $C_{n}$ be a cycle and $\overline{C_{n}}$ its complement such that $V\left(C_{n}\right)=\left\{x_{1}, x_{2}, \ldots, x_{n}\right\}$, $V\left(\overline{C_{n}}\right)=\left\{x_{1}^{c}, x_{2}^{c}, \ldots, x_{n}^{c}\right\}$ with the numbering of vertices in the natural order. Let $G(n)$ be the graph such that $V(G(n))=V\left(C_{n}\right) \cup V\left(\overline{C_{n}}\right)$ and $E(G(n))=E\left(C_{n}\right) \cup E\left(\overline{C_{n}}\right) \cup\left\{x_{i} x_{i}^{c} ; i \in\right.$ $\{1,2, \ldots, n\}\}$. Figure 21 shows an example of a $(2-d)$-kernel in $G(13)$. It is easy to check that if $n=5$, then $G(5)$ is isomorphic to the Petersen graph.

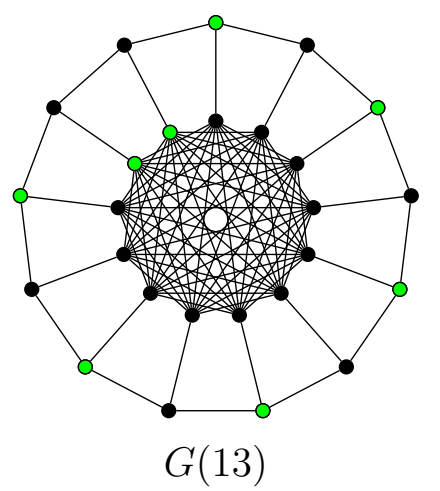

Figure 21. An example of a (2- $d)$-kernel in $G(13)$.

The next Theorem shows a complete characterization of graphs $G(n)$ with the (2- $d)$ kernel.

Theorem 2. Let $n \geq 5$ be integer. The graph $G(n)$ has a (2-d)-kernel if and only if $n$ is odd.

Proof. Let $n \geq 5$ be odd. We will show that $J=\left\{x_{2}^{c}, x_{3}^{c}, x_{1}, x_{4}, x_{6}, \ldots, x_{n-1}\right\}$ is the (2-d)kernel of the graph $G(n)$. The independence of $J$ is obvious. It is sufficient to show that $J$ 
is a 2-dominating set. By the definition of the graph $G(n)$, we can assume that $x_{n+1}=x_{1}$. Suppose that $y \in V(G(n)) \backslash J$. Hence, $y \in V\left(C_{n}\right)$ or $y \in V\left(\overline{C_{n}}\right)$. Let $y \in V\left(C_{n}\right)$. Thus $y=x_{k}, k \in\{2,3,5, \ldots, n\}$. If $x_{k}^{c} \notin J$, then there exist vertices $x_{k-1}, x_{k+1} \in J$ adjacent to $x_{k}$. If $x_{k}^{c} \in J$, then $k=2$ or $k=3$. For $k=2$, the vertex $x_{2}$ is adjacent to $x_{1}, x_{2}^{c} \in J$. Moreover, if $k=3$, then the vertex $x_{3}$ is adjacent to $x_{4}, x_{3}^{c} \in J$. Hence, every vertex from the set $V\left(C_{n}\right)$ is 2-dominated by the set $J$. Let now $y \in V\left(\overline{C_{n}}\right)$. Thus $y=x_{k}^{c}, k \in\{1,4,5, \ldots, n\}$. Then, the vertex $x_{k^{\prime}}^{c}, k \in\{5,6, \ldots, n\}$ is adjacent to $x_{2}^{c}, x_{3}^{c} \in J$. If $k=1$, then $x_{1}^{c} x_{1}, x_{1}^{c} x_{3}^{c} \in E(G(n))$. Moreover, for $k=4$ the vertex $x_{4}^{c}$ is adjacent to $x_{4}, x_{2}^{c}$. Therefore, vertices from the set $V\left(\overline{C_{n}}\right)$ are 2-dominated by $J$ and hence $J$ is a $(2-d)$-kernel of $G(n)$.

Conversely, suppose that a graph $G(n)$ has a $(2-d)$-kernel $J$. We will show that $n$ is odd. By the definition of the graph $G(n)$, we obtain that $J \cap V\left(\overline{C_{n}}\right) \neq \varnothing$. Otherwise, vertices from the set $V\left(\overline{C_{n}}\right)$ are not 2-dominated by the set $J$. Let $x_{1}^{c} \in J$. Then either $x_{2}^{c} \in J$ or $x_{n}^{c} \in J$. Otherwise, $x_{2}^{c}$ or $x_{n}^{c}$ is not 2-dominated. Hence, $\left|J \cap V\left(\overline{C_{n}}\right)\right|=2$. Without loss of generality assume that $x_{1}^{c}, x_{2}^{c} \in J$. This means that $x_{i}^{c}, i \in\{4,5, \ldots, n-1\}$ is 2-dominated by $J$ and $x_{3}^{c}, x_{n}^{c}$ are dominated by $J$. Let $J^{*}=J \backslash\left\{x_{1}^{c}, x_{2}^{c}\right\}$. Then, $J^{*} \subset V(C)$. Since $J$ is the (2- $d$ )-kernel, $x_{3}, x_{n} \in J^{*}$; otherwise, $x_{3}^{c}, x_{n}^{c}$ are not 2-dominated by $J$. Therefore, the graph $\left\langle\left\{x_{3}, x_{4}, \ldots, x_{n}\right\}\right\rangle_{G(n)} \cong P_{n-2}$ must have a $(2-d)$-kernel to 2-dominate vertices from $V\left(C_{n}\right) \backslash J^{*}$. This means that $n$ must be odd. Thus, $J^{*}=\left\{x_{3}, x_{5}, \ldots, x_{n}\right\}$, which ends the proof.

Finally, it turns out that if a graph $G(n)$ has $(2-d)$-kernel, then the number of $(2-d)$ kernels depends linearly on the number of vertices. Moreover, each (2- $d)$-kernel of $G(n)$ has the same cardinality.

Corollary 5. If $n \geq 5$ is odd, then $\sigma(G(n))=n$ and

$$
\gamma_{(2-d)}(G(n))=\Gamma_{(2-d)}(G(n))=\left\lfloor\frac{n}{2}\right\rfloor+2 .
$$

Proof. Let $n \geq 5$ be odd. From the construction of a (2-d)-kernel described in the proof of Theorem 2, we conclude that exactly two not adjacent vertices from the set $V\left(\overline{C_{n}}\right) \subset$ $V(G(n))$ belong to a (2- $d)$-kernel. The selection of these two vertices will determine the $(2-d)$-kernel in $G(n)$. Since two not adjacent vertices can be chosen on $n$ ways, $\sigma(G(n))=n$. Moreover, from the construction of $(2-d)$-kernels in $G(n)$, it follows that all of them have the same cardinality. Hence, $\gamma_{(2-d)}(G(n))=\Gamma_{(2-d)}(G(n))=\left\lfloor\frac{n}{2}\right\rfloor+2$, which ends the proof.

\section{Concluding Remarks}

In this paper, we considered two different generalizations of the Petersen graph, and we discussed the problem of the existence of $(2-d)$-kernels in these graphs. In particular, we determined the number of $(2-d)$-kernels in these graphs and their lower and upper $(2-d)$-kernel number. The generalized Petersen graphs considered in this paper are special cases of $I$-graphs (see, for example, [35]). The $I$-graph $I(n, j, k)$ is a graph with a vertex set $V(I(n, j, k))=\left\{u_{1}, u_{2}, \ldots, u_{n}, v_{1}, v_{2}, \ldots, v_{n}\right\}$ and an edge set $E(I(n, j, k))=\left\{u_{i} u_{i+j}, u_{i} v_{i}, v_{i} v_{i+k} ; i \in\{1,2, \ldots, n\}\right\}$, where subscripts are reduced modulo $n$. Because $P(n, k)=I(n, 1, k)$, the results obtained could be a starting point to studying and counting $(2-d)$-kernels in I-graphs. It could also be interesting to investigate the number of $(2-d)$-kernels in other generalizations of generalized Petersen graphs. For more generalizations, see, for example, [36].

Author Contributions: Both authors contributed equally to this work. All authors have read and agreed to the published version of the manuscript.

Funding: This research received no external funding.

Institutional Review Board Statement: Not applicable.

Informed Consent Statement: Not applicable. 
Conflicts of Interest: The authors declare no conflict of interest.

\section{References}

1. Diestel, R. Graph Theory, 3rd ed.; Springer: New York, NY, USA, 2005.

2. Morgenstern, O.; Von Neumann, J. Theory of Games and Economic Behavior; Princeton University Press: Princeton, NJ, USA, 1944.

3. Berge, C. Graphs and Hypergraphs; North-Holland Pub. Co.: Amsterdam, The Netherlands, 1973.

4. Berge, C.; Duchet, P. Perfect graphs and kernels. Bull. Inst. Math. Acad. Sin. 1988, 16, 263-274.

5. Berge, C.; Duchet, P. Recent problems and results about kernels in directed graphs. Discrete Math. 1990, 86, 27-31. [CrossRef]

6. Galeana-Sánchez, H.; Hernández-Cruz, C. On the existence of (k, l)-kernels in infinite digraphs: A survey. Discuss. Math. Graph Theory 2014, 34, 431-466. [CrossRef]

7. Galeana-Sánchez, H.; Hernández-Cruz, C.; Arumugam, S. On the existence of (k, l)-kernels in digraphs with a given circumference. AKCE Int. J. Graphs Comb. 2013, 10, 15-28.

8. Kucharska, M. On (k, 1)-kernel perfectness of special classes of digraphs. Discuss. Math. Graph Theory 2005, 25, 103-119. [CrossRef]

9. Włoch, I. On kernels by monochromatic paths in the corona of digraphs. Open Math. 2008, 6, 537-542. [CrossRef]

10. Bai, Y.; Fujita, S.; Zhang, S. Kernels by properly colored paths in arc-colored digraphs. Discrete Math. 2018, 341, 1523-1533. [CrossRef]

11. De la Maza, S.G.H.; Hernández-Cruz, C. On the complexity of the k-kernel problem on cyclically k-partite digraphs. Theoret. Comput. Sci. 2019, 795, 9-19. [CrossRef]

12. Hedetniemi, S.M.; Hedetniemi, S.T.; Knisely, J.; Rall, D.F.; Haynes, T.W. Secondary domination in graphs. AKCE Int. J. Graphs Comb. 2008, 5, 103-115.

13. Szumny, W.; Włoch, A.; Włoch, I. On the existence and on the number of $(\mathrm{k}, \mathrm{l})$-kernels in the lexicographic product of graphs. Discrete Math. 2008, 308, 4616-4624. [CrossRef]

14. Włoch, I. On kernels by monochromatic paths in D-join. Ars Combin. 2011, 98, 215-224.

15. Bednarz, U.; Włoch, I. On strong (1;1;2)-kernels in graphs. Ars Combin. 2020, 152, 32-43.

16. Bednarz, U. Strong (1;1;2)-kernels in the corona of graphs and some realization problems. Iran. J. Sci. Technol. Trans. A Sci. 2020, 44, 401-406. [CrossRef]

17. Bednarz, U.; Włoch, I. Fibonacci numbers in graphs with strong (1, 1, 2)-kernels. Bol. Soc. Mat. Mex. 2021, 27, 1-12. [CrossRef]

18. Michalski, A.; Włoch, I. On the existence and the number of independent (1,2)-dominating sets in the G-join of graphs. Appl. Math. Comput. 2020, 377, 125155. [CrossRef]

19. Fink, J.F.; Jacobson, M.S. n-domination in graphs. In Graph Theory with Applications to Algorithms and Computer Science; John Wiley \& Sons, Inc.: New York, NY, USA, 1985; pp. 283-300.

20. Włoch, A. On 2-dominating kernels in graphs. Australas. J. Combin. 2012, 53, 273-284.

21. Bednarz, P.; Włoch, I. An algorithm determining (2-d)-kernels in trees. Util. Math. 2017, 102, 215-222.

22. Bednarz, P.; Włoch, I. On (2-d)-kernels in the cartesian product of graphs. Ann. Univ. Mariae Curie-Skłodowska Sect. A 2016, 70, 1-8. [CrossRef]

23. Bednarz, P.; Hernández-Cruz, C.; Włoch, I. On the existence and the number of (2-d)-kernels in graphs. Ars Combin. 2015, 121, 341-351.

24. Bednarz, P. On (2-d)-Kernels in the Tensor Product of Graphs. Symmetry 2021, 13, 230. [CrossRef]

25. Nagy, Z.L. On the Number of k-Dominating Independent Sets. J. Graph Theory 2017, 84, 566-580. [CrossRef]

26. Gerbner, D.; Keszegh, B.; Methuku, A.; Patkós, B.; Vizer, M. An improvement on the maximum number of $k$-Dominating Independent Sets. J. Graph Theory 2019, 91, 88-97. [CrossRef]

27. Nagy, Z.L. Generalizing Erdős, Moon and Moser's result-The number of k-dominating independent sets. Electron. Notes Discrete Math. 2017, 61, 909-915. [CrossRef]

28. Behzad, A.; Behzad, M.; Praeger, C.E. On the domination number of the generalized Petersen graphs. Discrete Math. 2009, 308, 603-610. [CrossRef]

29. Ebrahimi, B.J.; Jahanbakht, N.; Mahmoodian E.S. Vertex domination of generalized Petersen graphs. Discrete Math. 2009, 309, 4355-4361. [CrossRef]

30. Yan, H.; Kang, L.; Xu, G. The exact domination number of the generalized Petersen graphs. Discrete Math. 2009, 309, $2596-2607$. [CrossRef]

31. Gabrovšek, B.; Peperko, A.; Žerovnik, J. Independent Rainbow Domination Numbers of Generalized Petersen Graphs P(n, 2) and $\mathrm{P}(\mathrm{n}, 3)$. Mathematics 2020, 8, 996. [CrossRef]

32. Erveš, R.; Žerovnik, J. On 2-Rainbow Domination Number of Generalized Petersen Graphs P(5k, k). Symmetry 2021, 13, 809. [CrossRef]

33. Watkins, M.E. A theorem on Tait colorings with an application to the generalized Petersen graphs. J. Combin. Theory 1969, 6 , 152-164. [CrossRef]

34. Alspach, B.; Liu, J. On the Hamilton connectivity of generalized Petersen graphs. Discrete Math. 2009, 309, 5461-5473. [CrossRef]

35. Boben, M.; Pisanski, T.; Žitnik, A. I-graphs and the corresponding configurations. J. Combin. Des. 2005, 13, 406-424. [CrossRef]

36. Saražin, M.L.; Pacco, W.; Previtali, A. Generalizing the generalized Petersen graphs. Discrete Math. 2007, 307, 534-543. [CrossRef] 